\title{
A Criterion for the Minimal Closedness of the Lie Subalgebra Corresponding to a Connected Nonclosed Lie Subgroup
}

\author{
JAN KUBARSKI
}

\begin{abstract}
A Lie subalgebra $h$ of a Lie algebra $\mathrm{g}$ is said to be minimally closed (after A. Malcev [11]) if the corresponding connected Lic subgroup is closed in the simply connected Lie group determined by $\mathrm{g}$. The aim of this paper is to prove the following theorem:

Let $H C G$ be any connected (not necessarily closed) Lie subgroup of a Lie group $G$. Denote by, $h, \bar{h}$ and $\mathrm{g}$ the Lie algebras of $H$, of its closure $H$ and of $G$, respectivell: If there exists a Lie subalgebra $\mathrm{c} \subset \mathrm{g}$ wuch that $(a) \mathrm{c}+\overline{\mathrm{h}}=\mathrm{g}$. (b) $\mathrm{c} \cap \overline{\mathrm{h}}=\mathrm{h}$, then $\mathrm{h}$ is minimally closed.
\end{abstract}

As a coroliary we obtain that if $\pi_{1}(G)$ is finite, then no such a Lic subalgebra $c$ exists provided that $H$ is nonclosed.

The prool is carried out on the ground of the theory of Lie algebroids and by using some ideas from the theory of transwersally complete foliations.

\section{INTRODUCTION}

A) Let $G$ be any connected lie group. Assume that $H \subset G$ is any of its connected and nonclosed Lie subgroups. Denote by $h, \bar{h}, g$ the l.ie algebras of $H$, of its closure $\bar{H}$ and of $G$, respectively.

0.1. Problem. Does there exist a Lie subalgebra $c \subset g$ such that (a) $c+\bar{h}=g .(b) c \cap \bar{h}=h$ ?

In work [7], some topological obstructions of the existence of such a lie algebra $c$ were found. Namely, the following theorem was proved. 
0.2. Theorem. If the following homomorphism of algebras

$$
V(\overline{\mathrm{h}} / \mathrm{h})^{*} \longrightarrow\left(V \overline{\mathrm{k}}^{*}\right)_{l} \stackrel{h_{p}}{\longrightarrow} H_{\mathrm{dR}}(G / \bar{H})
$$

(where $h_{P}$ is the Chern-Weil homomorphism of the $\bar{H}$-principal fibre bundle $P=(G \rightarrow G / \bar{H}))$ is nontrivial, then such a Lie subalgebra $c$ does not exist.

Next, it was noticed that the case of a compact and semisimple Lie group is a case for which homomorphism (1) is always nontrivial. As a corollary we have.

0.3. Theorem. If $G$ is a compact and semisimple Lie group, then no Lie subalgebra c fulfilling (a) and (b) above exists.

We add that (1) appears as the Chern-Weil homomorphism of the Lie algebroid of the TC-foliation $\mathscr{F}=\{g H ; g \in G\}$ of left cosets of $G$ by $H$, determined by the author [7], [8].

B) In the present paper, a Lie algebroid of a connected (not necessarily closed) Lie subgroup $H$ of a given Lie group $G$ is constructed precisely. It can be noticed that it is the same as the one constructed in the theory of P. Molino [12] for the corresponding TC-foliation $\mathscr{F}$ of left cosets. Next, we get to the core of the structure of this Lie algebroid and prove some strengthening of theorem 0.3 (by weakening the assumptions to the finiteness of $\pi_{1}(G)$ ) without using any characteristic classes. This fact is obtained as a corollary from the theorem saying that:

0.4. Theorem. The existence of a Lie subalgebra c fulfilling (a) and (b) above implies the minimal closedness of $\mathbf{h}$ (in the sense of Malcev [11]).

\section{PRELIMINARIES}

We give a few elementary facts concerning the theory of Lic algebroids; needed in the sequel. We assume that in our paper all the manifolds considered are of $C^{\infty}$-class and Hausdorff. By $\Omega^{\circ}(M)$ we denote the ring of $C^{\infty}$ functions on a manifold $M$, by $\mathcal{X}(M)$ the Lie algebra of $C^{\infty}$ vector fields on $M$, and by $\operatorname{Sec} A$ the $\Omega^{\circ}(M)$-module of all $C^{\infty}$ global cross-sections of a given vector bundle $A$ (over $M$ ).

1.1. Definition [15], [16]. By a transitive Lie algebroid on a manifold $M$ we mean a sustem

$$
A=(A, \llbracket[\cdot, \cdot] \cdot \gamma)
$$


consisting of a vector bundle $A$ (over $M$ ) and mappings

$$
\llbracket \cdot \cdot \rrbracket: \operatorname{Sec} A \times \operatorname{Sec} A \rightarrow \operatorname{Sec} A, \quad \gamma: A \rightarrow T M,
$$

such that

(i) $(\operatorname{Sec} A, \mathbb{I} \cdot, \cdot \mathbb{\Pi})$ is an $\mathbf{R}$-Lie algebra,

(ii) $\gamma$, called by $\mathrm{K}$. Mackenzie [9] an anchor, is an epimorphism of vector bundles, bras,

(iii) $\operatorname{Sec} \gamma: \operatorname{Sec} A \longrightarrow \mathrm{X}(M), \xi \rightarrow \gamma^{\prime} \xi$, is a homomorphism of lie alge-

(iv) $\llbracket \xi, f \cdot \eta \rrbracket=f^{\prime} \cdot \llbracket \xi, \eta \rrbracket+\left(\gamma^{\prime \prime} \xi\right)(f) \cdot \eta$ for $f \in \Omega^{\prime \prime}(M), \xi, \eta \in \operatorname{Sec} A$.

$\mathrm{g}:=\operatorname{Ker} \gamma$ is a vector bundle and the short exact sequence

$$
\mathrm{O} \rightarrow \mathrm{g} \subset A \stackrel{\gamma}{\longrightarrow} 7 M \longrightarrow \mathrm{O}
$$

is called an Aligah sequence of (2); in each vector space $\mathbf{g}_{\mid x}=\operatorname{Ker} \gamma_{\mid x}, x \in M$. some Lie algebra structure is defined by

$$
[v, w]:=\llbracket \xi, \eta \rrbracket(x), \quad \xi, \eta \in \operatorname{Sec} A, \quad \xi(x)=v, \quad \eta(x)=w, \quad v, w \in \mathbf{g}_{1} .
$$

$\mathbf{g}_{\mid x}$ is called the isorropy Lie algebra of (2) at $x . \mathbf{g}$ is a Lie algebra bundle [2]. [5], [6]. [9] called (after Mackenzic) the adioint of (2).

Let (2) and $\left(A^{\prime}, \mathbb{[} \cdot, \cdot \mathbb{I}^{\prime}, \gamma^{\prime}\right)$ be two transitive Lie algebroids on the same manifold $M$. By a strong homomorphism

$$
H:\left(A^{\prime}, \llbracket \cdot \cdot, \mathbb{I}^{\prime}, \gamma^{\prime}\right) \rightarrow(A, \mathbb{\llbracket} \cdot, \cdot \mathbb{\rrbracket}, \gamma)
$$

between them [4], [10, p. 273] we mean a strong homomorphism of vector bundles $H: A^{\prime} \longrightarrow A$, such that

(i) $\gamma_{0} H=\gamma^{\prime}$,

(ii) $\operatorname{Sec} H: \operatorname{Sec} A^{\prime} \rightarrow \operatorname{Sec} A, \xi \longrightarrow H \circ \xi$. is a homomorphism of Lie alge-, bras.

If homomorphism (4) is a bijection, then $H^{-1}$ is also a homomorphism of Lie algebroids; then $H$ is called an isomorphism of Lie algebroids. 
1.2. Example. By a trivia/ Lie algebroid [14] we mean any algebroid isomorphic to $\left.(T M \times \mathfrak{g}, \mathbb{I} \cdot, \cdot], p r_{1}\right)$ where $\mathrm{g}$ is a finitely dimensional Lie algebra and the bracket $[$.,$:$ ] $]$ is defined by

$$
\|(X, \sigma),(Y, \eta) \rrbracket=\left([X, Y], Y_{X} \eta-Y_{\gamma} \sigma+[\sigma, \eta]\right)
$$

$X, \quad Y \in X(M), \quad \sigma, \eta: \quad M \rightarrow g \quad([\sigma, \eta]$ is defined point by point: $[\sigma, \eta](x)=[\sigma(x), \eta(x)], x \in M)$.

1.3. Example (See [5], [6], [9]). By the Lie algebroid $A(P)$ of $a$ principal fibre bundle $P=(P, \pi, M, G,$.$) we mean a transitive Lie algebroid$ on $M\left(A(P),[[\cdot, \cdot], \gamma)\right.$ in which $A(P)=T P / G, \gamma([v])=\pi_{*}(v)$ where $[v]$ denotes the equivalence class of $v$, and the bracket $[\xi \xi, \eta], \xi, \eta \in \operatorname{Sec} A(P)$, is constructed on the basis of the following observation: For each cross $\rightarrow$ section $\eta \in \operatorname{Sec} A(P)$, there exists exactly one $C^{\infty}$ right-invariant vector field $\eta^{\prime} \in X^{R}(P)$ such that $\left[\eta^{\prime}(z)\right]=\eta(\pi z)$, and the mapping $\operatorname{Sec} A(P) \longrightarrow X^{R}(P), \eta \longrightarrow \eta^{\prime}$, is an isomorphism of $\Omega^{\circ}(M)$-modules. The bracket $\llbracket \xi, \eta \rrbracket$ is a cross-section of $A(P)$ such that $\llbracket \xi \xi, \eta \rrbracket^{\prime}:=\left[\xi, \eta^{\prime}\right]$.

The Lie algebroid of a trivial principal fibre bundle $P=M \times G$ is canonically isomorphic to the trivial Lic algebroid $A=T M \times \mathrm{g} . \mathrm{g}$ is the right Lie algebra of $G$, via

$$
A(P)=T(M \times G) / G=T M \times(T G / G) \ni(v,[w]) \longrightarrow\left(v, \theta^{k}(w)\right) \in T M \times g ;
$$

$(\omega)$ denotes the canonical right-invariant 1 form on $G[5],[6]$.

A transitive Lie algebroid strongly isomorphic to $A(P)$ for some principal libre bundle is called integrable [9]. There exist non-integrable Lie algebroids discovered by R. Almeida and P. Molino [1]. Lic algebroids of some TCfoliations are non-integrable, for example, the Lie algebroid of the foliation of left cosets of any connected and simply connected Lie group by a connected nonclosed Lie subgroup has this property.

1.4. Definition. By a connection in transitive Lie algebroid (2), see [5], [9]. [15], we mean a homomorphism of vector bundles $\lambda: T M \rightarrow A$ such that $\gamma \circ \lambda=i d_{T, A}$, i.e. a splitting of Atiyah sequence (3) of $A$

$$
\mathrm{O} \longrightarrow \mathrm{g}=\stackrel{\frac{\gamma}{\longrightarrow}}{\rightarrow} T M \rightarrow 0 \text {. }
$$

By a curvalure tensor of a connection $\lambda$ in (2) we shall mean a tensor $\Omega_{f} \in \Omega^{2}(M ; \mathbf{g})\left(=\operatorname{Sec}^{2} T^{*} M \otimes \mathbf{g}\right)$ defined by

$$
\Omega_{b}(X, Y)=\lambda[X, Y]-\llbracket \lambda X, \lambda Y \rrbracket, X, Y \in X(M) .
$$


$\lambda$ also determines a covariant derivative $\nabla$ in $\mathbf{g}$ by

$$
\nabla_{X} \sigma=\llbracket \lambda X, \sigma \rrbracket . \quad X \in X(M), \quad \sigma \in \operatorname{Sec} \mathbf{g},
$$

See [5], [9].

It turns out that the Lie algebra structure in $\operatorname{Sec} A$ is uniquely determined by $\mathbf{g}, \nabla, \Omega_{h}$ and $\lambda$, namely, we have

1.5. Theorem [5], [9]. The mapping $\varphi: T M \oplus \mathrm{g} \rightarrow A,(v, w) \rightarrow \lambda v+w$, is an isomorphism of Lie algebroids provided that in $T M \oplus \mathrm{g}$ the following Lie algebroid structure is defined:

(a) the bracket:

$\llbracket(X, \sigma), \quad(y, \eta) \rrbracket=\left([X, Y],-\Omega_{h}(X, Y)+\nabla_{X} \eta-\nabla_{Y} \sigma+[\sigma, n]\right), \quad X, Y \in X(M)$, $\sigma, n \in \operatorname{Sec}([\sigma, \eta]$ is defined point by point: $[\sigma, \eta](x)=[\sigma(x), \eta(x)], x \in M)$.

(b) the anchor: $\gamma=p r_{1}: T M \oplus \mathbf{g} \longrightarrow 7 M$.

\section{THE LIE ALGEBROID OF A CONNECTED (NOT NECESSARILY CLOSED) LIE SUBGROUP}

Let $G$ be any connected Lie group and $H \subset G$ any connected (not necessarily closed) Lic subgroup of $G . H$ determines the foliation $=\{g H$ : $g \in G\}$ of left cosets of $G$ by $H$. [13] because right-invariant vector fields are from the normalizer of $X_{(\%)}$ and generate the entire tangent space $T_{g} G$ for any $g \in G$.

Denote by $E$ the tangent bundle to $\%$ and $Q=T G / E \stackrel{r}{\longrightarrow} G$ the transversal bundle of . Let

$$
\alpha: T G \rightarrow Q
$$

be the canonical projection and let $\bar{v}, v \in T G$, denote the vector $\alpha(v)$. $R_{l}: T G \longrightarrow T G$ stands for the differential of the right translation by $t \in G$.

2.1. Lemma. (i) $R_{t}, t \in \bar{H}$ ( $\bar{H}$ is the closure of $H$ ), maps $E$ into $E$ inducing the isomorphism of vector bundles $\bar{R}_{l}: Q \rightarrow Q, \bar{v} \mapsto \overline{R_{1}(v)}$.

(ii) The mapping $\bar{R}: Q \times \bar{H} \longrightarrow Q,(\bar{v}, 1) \mapsto \bar{R}_{i}(\bar{v})$, is a right strongly free action. 
Proof. Easy calculations.

As a corollary we obtain

2.2. The topological space $A(G ; H)$ of orbits of the action $\bar{R}$, i.e.

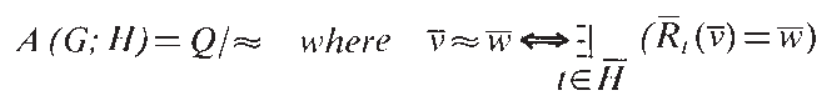

has a uniquely determined structure of a $C^{\infty}$ manifold, such that the canonical projection $\beta: Q \longrightarrow A(G ; H)$ is a submersion.

In the sequel, the vector $\beta(\vec{v}), \bar{v} \in Q$, will be denoted by [i]] and $\pi_{b}: G \longrightarrow G / \bar{H}$ stands for the canonical projection. Of course, $\bar{\gamma}: A(G$; $H) \rightarrow G / \vec{H},[\bar{w}] \mapsto \pi_{h}(r \bar{w})$, is a correctly defined projection. Its smoothness follows immediately from the commutativity of the diagram

$$
\begin{aligned}
& Q \stackrel{\beta}{\rightarrow} A(G ; H) \\
& \stackrel{\downarrow}{r} \pi_{b} G / \frac{b^{r}}{H}
\end{aligned} .
$$

For the fibre $A(G ; H)_{\mid \bar{y}}$ of $\bar{r}$ over $\bar{g} \in G / \bar{H}$, the mapping $\beta_{k}: Q_{\mid k} \rightarrow A(G ; H)_{\mid \bar{g},}$ $g \in \pi_{b}{ }^{-1}(\bar{g})$, is a bijection. Via $\beta_{k}$ we introduce in $A(G ; H)_{\mid \bar{g}}$ some structure of a real vector space and, clearly, it is independent of the choice of $g$. We wish to arrange the system $(A(G, H), \bar{r}, G / H)$ to be a vector bundle. For the purpose, we find local trivializations of this system.

2.3. Definition. $A C^{\infty}$ cross-section $\zeta \in S e c Q$ is called a transversal field if, for any $g \in G$ and $t \in \bar{H}$,

$$
\zeta(g t)=\bar{R}_{1}(\zeta(g))
$$

(that is, if $\zeta$ is $\bar{H}$-right-invariant).

2.4. Example. The $C^{\infty}$ cross-section $\bar{Y}_{n}:=\alpha " Y_{w}$ where $Y_{w}$ stands for the right-invariant vector field on $G$ generated by $w \in g$ ( $g$ is the Lic algebra of $G$ ) is a transversal field. Therefore, transversal fields generate the entire space $Q_{l g}$ for any $g \in G$.

2.5. Remarks. Denote by $/(G ; H)$ the space of all transversal fields.

(a) $/(G ; H)$ forms a module over the ring $\Omega^{\circ}(G / \bar{H})$ under the multiplication $\bar{f} \cdot \zeta:=\bar{f} \cdot \pi_{t} \cdot \zeta, \bar{f} \in \Omega(G / \bar{H}) . \zeta \in /(G ; H)$. 
(b) If transversal fields $\zeta_{1}, \ldots, \zeta_{x}$ are linearly independent at a point $g \in G$, then, immediately by the definition, they are linearly independent at each point $g t, t \in \bar{H}$, and, in consequence, at some open $\bar{F}$-saturated open subset where $\mathscr{F}_{b}$ is the so-called basic foliation $\mathscr{F}_{b}=\{g \vec{H} ; g \in G\}$.

(c) Let $\zeta, \zeta_{i} \in l(G ; H), i \leq s$. If $\zeta_{i}$ are linearly independent on $U=\pi_{b}{ }^{-1}[\bar{U}]$ ( $\bar{U}$ open in $G / \bar{H})$ and $\zeta=\sum_{i=1}^{i} f^{i} \zeta_{i}$ for $f^{i} \in \Omega^{\circ}(U)$, then the functions $f^{i}$ are of the form $f^{i}=\vec{f}^{i}{ }, \pi_{b} \mid U$ for some $\vec{f}^{i} \in \Omega^{*}(\bar{U})$.

2.6. Proposition. Let $q=\operatorname{dim} G-\operatorname{dim} H$, i.e. $q=$ codim $J^{-}$. Suppose that $\zeta_{1}, \ldots, \zeta_{q}$ are transversal fields linearly independent at each point of a set $U=\pi_{h}{ }^{-1}[\bar{U}], \bar{U}$ open in $G / \bar{H}$. Then

$$
\begin{aligned}
& \varphi: \bar{U} \times \mathbf{R}^{y} \longrightarrow \bar{r}^{-1}[\bar{U}] \subset A(G: H) \\
& (\bar{g}, \alpha) \rightarrow\left[\Sigma \alpha^{i} \zeta_{i}(g)\right], g \in \pi_{h}^{-1}(\bar{g}),
\end{aligned}
$$

is a local trivialization of $\bar{r}: A(G ; H) \rightarrow G / \bar{H}$.

Proof. Of course, $\varphi_{\bar{g}}: \overrightarrow{\mathbf{R}}^{q} \longrightarrow A(G ; H)_{\mid \bar{g}}, \bar{g} \in \vec{U}$, is an isomorphism of vector spaces. This proposition will be proved by showing that $\varphi$ is a diffeomorphism. For the purpose, take the mapping $\psi: U \times \mathbf{R}^{q} \longrightarrow r^{-1}[U] \subset Q$, $(g, \alpha) \rightarrow \sum \alpha^{j} \zeta_{i}(g)$, being a local trivialization of $Q$. Our assertion follows now from the commutativity of the diagram

$$
\begin{gathered}
U \times \mathbf{R}^{\prime \prime} \stackrel{\psi}{\cong} r^{-1}[U] \subset Q \quad \stackrel{r}{=} G \\
\downarrow \pi_{h} \times i d d \quad \\
\bar{U} \times \mathbf{R}^{4} \stackrel{\varphi}{\rightarrow} \bar{r}^{-1}[\bar{U}] \subset A(G ; H) \stackrel{\bar{r}}{=} G / \bar{H}
\end{gathered}
$$

2.7. Remark. The structure of a $C^{\infty}$ manifold in $A(G ; H)$ can be obtained independently by demanding that $\varphi^{\prime} s$ be diffeomorphisms.

Now, we introduce a structure of a Lie algebroid into the vector bundle $A(G ; H)$. Firstly, we define the anchor $\gamma: A(G ; H) \rightarrow T(G / \bar{H})$ by $\left[\bar{w}^{*}\right] \rightarrow \pi_{b^{*}}(w)$ (the correctness is easy to obtain). Secondly, we introduce in $\operatorname{Sec} A(G ; H)$ a structure of a Lie algebra in the way described below. 
Take a homomorphism of $\Omega^{\circ}(G / \bar{H})$ - modules

$$
\left(\because I(G ; H) \longrightarrow \operatorname{Sec} A(G ; H), \zeta \rightarrow c_{\zeta},\right.
$$

where $c_{\zeta}$ is a $C^{\infty}$ cross-section of $A(G: H)$ defined by $c_{\zeta}(\bar{g})=[\zeta(g)]$, $g \in \pi_{b}{ }^{-1}\left(\frac{g}{g}\right)$.

2.8. Lemma. cis an isomorphism of $\Omega^{\circ}(G / \bar{H})$-modules.

Proof. We check at once that (5) is a monomorphism. To see that it is also an epimorphism, take an arbitrary $C^{\infty}$ cross-section $\xi \in \operatorname{Sec} A(G ; H)$ and define a cross-section $\zeta$ of $Q$ in such a way that the diagram

$$
\begin{aligned}
& Q \stackrel{\beta}{\longrightarrow} A(G ; H) \\
& \zeta \underset{\pi_{h}}{\longrightarrow} G / \bar{H}
\end{aligned}
$$

commutes, i.e. $c_{\xi}=\xi$. The smoothness of $\zeta$ is the last thing to notice. In order to get this, take transversal fields $\zeta_{1}, \ldots, \zeta_{4}$ being a basis on $U=\pi_{h}{ }^{-1}[\bar{U}]$ ( $\bar{U}$ is open in $G / \bar{H}$ and contains an arbitrarily taken point of $G / \bar{H})$. Then $c_{\zeta_{1}}, \ldots, c_{c_{2}}$ forms a basis of $A(G ; H)$ on $\bar{U}$. Therefore, $\xi=\sum \bar{f}^{i} c_{\xi_{i}}$ on $\bar{U}$ for some $\bar{f}^{\prime} \in \Omega^{\prime}(\bar{U})$. Of course, $\zeta=\Sigma \bar{f}^{i}{ }^{\prime} \pi_{h} \cdot \zeta_{i}$ on $U$, which ends the proof.

2.9. The space $I(G ; H)$ has a natural structure of a real lie algebra. Indeed, let $\zeta, v \in l(G ; H) \subset$ Sec $Q$. Take arbitrary yector fields $X, Y \in \mathrm{X}(G)$ such that $\zeta=\bar{X}(:=\alpha \cdot X)$ and, analogously, $v=\bar{Y}$. Put.

$$
[\zeta, v]:=\overline{[X, Y]} .
$$

We need notice that

(a) $\overline{[X, Y]} \in /(G ; H)$.

(b) definition (6) is correct.

L.et us first observe 
2.10. Lemma. If $\zeta \in l(G ; H)$ is of the form $\zeta=\bar{X}$ for a vector field $X \in \mathrm{X}(G)$, then $X$ belongs to the normalizer of $\mathrm{X}(\mathscr{F})$, that is,

$$
[X, Y] \in X(S) \text { for all } Y \in X(S F)
$$

[i.e. $X$ is the so-called foliate vector field for $\mathscr{F}$, see [13]].

Proof. Of course, it is sufficient to show relation (7) for left-invariant vector fields $Y=X_{h}, h \in \mathbf{k}$, only. To this end, take an arbitrary $g \in G$ and express $\zeta$ locally on a set $\left.U=\pi_{b}-\mid \bar{U}\right]$ containing $g(\bar{U}$ open in $G / \bar{H})$, in the form $\zeta_{\mid U}=\Sigma \bar{f}^{i}{ }^{\prime} \pi_{b \mid U} \cdot \bar{Y}_{w_{i} \mid U}, \bar{f}^{i} \in \Omega(\bar{U}), w_{i} \in \Omega$ (for $\bar{Y}_{w}$, see 2.4). Then

$$
Z:=\sum \bar{f}^{i}{ } \pi_{h \mid U} \cdot Y_{M_{i} \mid U}-X_{\mid U} \in \mathbf{X}\left(\mathscr{F}_{U}\right)
$$

and, furthermore, we have

$$
\begin{aligned}
{\left[X, X_{h}\right]_{\mid U} } & =\left[\Sigma \bar{f}^{i} \pi_{h \mid U} \cdot Y_{w ; \mid U}-Z, X_{h \mid U]}\right] \\
& =-\left[Z, X_{h}\right]_{\mid U} \in X\left(\mathscr{F}_{U}\right),
\end{aligned}
$$

thus $\left[X, X_{h}\right] \in X(\mathscr{Y})$.

2.11. Remark. It can be proved that condition (7) is equivalent to the fact that $\zeta:=\bar{X}$ is a transversal field; however, the sufficiency of this condition will not be used in the sequel.

Now, we are able to prove (a) and (b) from 2.9.

(a): To get the equality $\bar{R}_{i}(\overline{[X, Y]}(g))=\overline{[X, Y]}(g t), g \in G, t \in \bar{H}$, take the vector fields $Z_{1}=R_{t} X-X$ and $Z_{2}=R_{1} Y-Y$ tangent to $\mathscr{F}$. Applying 2.10, we deduce that

$$
\begin{aligned}
\bar{R}_{t}(\overline{[X, Y]}(g)) & =\overline{R_{l}([X, Y](g))}=\overline{R_{t}([X, Y])(g t)} \\
& =\overline{\left[R_{t} X, R_{t} Y\right](g t)}=\overline{\left[X+Z_{1}, Y+Z_{2}\right]}(g t) \\
& =\overline{[X Y]}(g t) .
\end{aligned}
$$

(b): Immediately from 2.10 .

2.12. In $\operatorname{Sec} A(G ; H)$ we introduce the bracket $\llbracket . . . \rrbracket$ (forming a Lie algebra) by demanding that (5) be an isomorphism of Lie algebras, i.e. $\llbracket c_{\zeta}, c_{n}, \rrbracket:=c_{\mid \zeta, v]}, \zeta, v \in l(G ; H)$. 
2.13. Theorem. The system

$$
A(G ; H)=(A(G ; H), \mathbb{I} \cdot, \mathbb{\rrbracket}, \gamma)
$$

is a ransitive Lie algebroid on $G / \bar{H}$.

Proof. (1) $\operatorname{Sec} \gamma: \operatorname{Sec} A(G ; H)-\mathcal{H}(G / \bar{H})$ is a homomorphism of Lie algebras. To see this, take $\xi, \eta \in \operatorname{Sec} A(G ; H)$. Find vector fields $X, Y \in X(G)$ such that $\xi=c \bar{x}, \eta=c \bar{\gamma}$. By the definition of $\gamma$.

$$
(\operatorname{Soc} \gamma)\left(c_{\bar{x}}\right)(\bar{g})=\pi_{h * g}\left(X_{b}\right) \text { for } \bar{g}=\pi_{h}(g), g \in G,
$$

from which we obtain that $X$ is $\pi_{l}$-related to $\gamma$ " $\xi$ and, analogously, $Y$ to $\gamma$ an Therefore $[X, Y]$ is $\pi_{b}$-related to $[\gamma \cdot \xi, \gamma n \eta]$ and to $\left.\gamma^{\prime \prime} \llbracket \xi, \eta\right]$ simultaneously. which confirms our assertion.

(2) The equality $\|\xi, \bar{f} \cdot \eta\|=\bar{f} \cdot\|\xi, \eta\|+(\gamma, \xi)(\bar{f}) \cdot \eta, \quad \bar{f} \in \Omega^{\circ}(G / \bar{H})$, $\xi, \eta \in \operatorname{Sec} A(G ; H)$, follows easily from

$$
\left[\bar{X}, \bar{f} \cdot \pi_{b} \cdot \bar{Y}\right]=\bar{f} \cdot \pi_{b} \cdot[\bar{X}, \bar{Y}]+(\gamma \cdot c \bar{x})(\bar{f}) \cdot \bar{Y}
$$

Lie algebroid (8) will be called the Lie algebroid of a Lie subgroup $H$ of $G$. It can be interesting only in the case of a nonclosed $H$ because the closedness of $H$ implies the triviality of $A(G ; H): A(G ; H) \cong T(G / \bar{H})$.

2.14. Remark. One can prove [cf. [7]] that Lic algebroid (8) is equal to the one constructed by P. Molino [12], [13] for the TC-foliation

\section{STRUCTURE THEOREMS}

Let $(8)$ be the Lie algebroid of a connecled Lie subgroup $H$ of a connected Lic group $G$ and

$$
O \longrightarrow \mathrm{g} \longrightarrow A(G ; H) \stackrel{\gamma}{\longrightarrow} T(G / \bar{H})-O
$$

its Atiyah sequence. In this section we prove three fundamental facts concerning $A(G ; H)$ :

- The adjoint Lie algebra bundle $\mathrm{g}$ of $A(G ; H)$ is a rivial bundle of abelian Lie algebras.

- If the Lie algebroid $A(G ; l l)$ admits a flat connection (i.t. a connection with the zero (curvature sensor), then it is trivial. 
- Let $\mathbf{h}, \overline{\mathbf{h}}, \mathbf{g}$ denote the Lie algebras of $H, \bar{H}$ and $G$, respectivety. Suppose that there exists a Lie subalgebra $\subset \subset g$ such that (a) $\mathrm{c}+\overline{\mathbf{h}}=\mathrm{g}$, (b) $\mathrm{c} \cap \overline{\mathbf{h}}=\mathbf{h}$. Then $A(G ; H)$ admits a flat connection.

The crucial role in the proving of the first fact is played by the following Malcev theorem (for a short "foliated" proof of it, see [7]).

3.1. The Malcev Theorem [11], [17]. If $H$ is a dense connected Lie subgroup of a Lie group $T$, then $H$ is a normal subgroup of $T$ and $T / H$ is abelian.

By this, according to our notations, $h$ is an ideal of $\bar{h}$ and $\bar{h} / \boldsymbol{h}$ is an abelian Lie algebra.

3.2. Theorem. For a vector $w^{\in} \in \overline{\mathrm{h}}$. the cross-section $\bar{X}_{1,}$ of the transversal bundle $Q$. induced by the left-invariant vector field $X_{n}$, is a transversal field. and the mapping

$$
\varphi: G / \bar{H} \times \overline{\mathrm{h}} / \mathrm{h} \rightarrow \mathrm{g} .(\bar{g},[w]) \rightarrow\left[\bar{X}_{w}(g)\right], g \in \pi_{h}^{-1}(\bar{g}),
$$

is a global trivialization of the Lie algebra bundle $\$$

Proof. It is sufficient to show that $\bar{X}_{w}, w \in \bar{h}$, is a transversal field; the rest is easy. Clearly, for $t \in \bar{H}$ and $g \in G \quad \bar{R}_{f}\left(\bar{X}_{n}(g)\right)=\bar{L}_{g}\left(\bar{R}_{f}(w)\right)$ and $\bar{X}_{w}(g t)=\bar{L}_{g}\left(\bar{L}_{t}(w)\right)$ where $\bar{L}_{g}: Q \longrightarrow Q$ is an automorphism of the vector bundle $Q$. determined by the differential $l_{z_{k}}$ of the left-translation by $g$. Therefore, it remains to prove that $R_{1}\left(w^{\prime}\right)-L_{1}(w) \in E_{1}$, which means that the vector field $X:=Y_{w}-X_{w}$ is tangent to the foliation at each point of $\bar{H}$.

Firstly, we notice that $X$ is foliate; to see this, we calculate: Let $h \in \mathfrak{h}$, then $\left[X, X_{h}\right]=\left[Y_{w}-X_{w}, X_{h l}\right]=X_{\mid h, w]} \in X\left(y^{\prime}\right)$ because $[h, w] \in h$ according to the Malcev theorem.

Secondly, any foliate vector field $X$ (for a foliation $y^{;}$) in any distinguished local coordinates $x=\left(x^{1}, \ldots, x^{\prime \prime}, y^{1}, \ldots, y^{4}\right)(p=d i m, \%$.

$q=$ codim $y(x)$ is of the form $x(x, y)=\sum a^{i}\left(x, y^{\prime}\right) \frac{\partial}{\partial x^{i}}+\Sigma b^{\prime}(y) \frac{\partial}{\partial y^{j}}[13]$ therefore, which is easy to see, if it is tangent to $\%$ at a point $z$, then it is tangent to $Y$ at each point of the closure of the leaf through $z$. In our situation. $X(e)=\left[Y_{w}-X_{w}\right](e)=O \in E_{\mid e}$, so - by the above our theorem is proved.

Now, we proceed to the second problem. 
3.3. Theorem. If' the Lie algebroid $A(G ; H)$ is flat, then it is trivial.

Proof. Let $\lambda: T(G / \bar{H}) \rightarrow A(G ; H)$ be a flat connection in $A(G ; H)$. Then, taking account of 1.4 and isomorphism (9) of Lie algebra bundles, we have an isomorphism of Lie algebroids

$$
\rho: T(G / \bar{H}) \times \overline{\mathrm{h}} / \mathrm{h} \rightarrow A(G ; H),(v,[w]) \rightarrow \lambda v+\left[\bar{X}_{w}(g)\right],
$$

$v \in T_{\pi} G / \bar{H}, \bar{g}=\pi_{b}(g), g \in G$, provided that in $T(G / \bar{H}) \times \bar{h} / \mathrm{h}$ the Lie algebroid structure is defined by the following formula

$$
\llbracket(X, \sigma),(Y, \eta)]=\left([X, \gamma], \nabla_{X}^{\prime} \eta-\nabla_{Y}^{\prime \prime} \sigma+[\sigma, \eta]\right),
$$

$X, Y \in X(G / \bar{H}), \sigma, \eta: G / \bar{H} \rightarrow \overline{\mathrm{h}} / \mathrm{h}$, where $\nabla^{\circ}$ is a covariant derivative in the trivial vector bundle $T(G / \bar{H}) \times \overline{\mathrm{h}} / \mathrm{h}$, such that $\varphi$ maps $\nabla^{\prime \prime}$ onto $\nabla$, i.e.

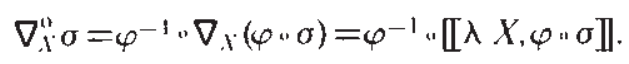

Looking at example 1.2, we see that to end the prool, it is sufficient to show the equality

$$
\nabla_{x}^{\prime \prime}=y_{x}, \quad X \in \mathrm{X}(G / \bar{H}),
$$

which is equivalent to the fact that the covariant derivative $\nabla_{x}^{0}$ of any constant function $\hat{w}: G / \bar{H} \longrightarrow \bar{h} / \mathrm{h}, \bar{g} \longrightarrow[w], w \in \bar{h}$, is zero, i.e. that $\llbracket \lambda X, c_{x_{w}} \rrbracket=0$. The cross-section $\lambda X$ is locally of the form $\lambda X=\Sigma \bar{f}^{\prime} c_{r_{w ;}}$, $\bar{f}^{i} \in \Omega^{\prime \prime}(G / \bar{H})$, thus

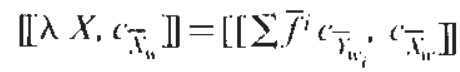

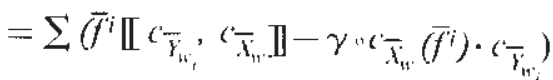

$$
\begin{aligned}
& =0
\end{aligned}
$$

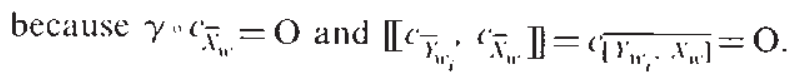

It remains to consider the third problem.

3.4. Theorem. Suppose that there exists a Lie subalgebra $\subset \subset \mathrm{G}$ such that (a) $\mathrm{c}+\overline{\mathrm{h}}=\mathrm{g}$. (b) $\mathrm{c} \cap \overline{\mathrm{h}}=\mathrm{h}$. Then $A(G ; H)$ admits a fiat connection.

Proof. The construction of a flat connection in $A(G ; H)$ has four steps. 
Step 1. Denote by $\bar{C} \subset T G$ the left-invariant distribution generated by $c$. i.e. the vector bundle tangent to the foliation $\{g F, g \in G\}$ where $F$ is the connected Lie subgroup with the Lie algebra equalling $c$. $\bar{C}$ fullils the following conditions (in which $E_{b}$, is the vector bundle tangent to the foliation $\left.\breve{S}_{\mathrm{b}}=\{g \bar{H} ; g \in G\}\right)$ :

(I) $\bar{C}+E_{h}=T G$.

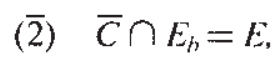

( $\overline{3}) \quad \bar{C}$ is $\bar{H}$-right-invariant [i,e, $\left.\bar{C}_{\mid k t}=R_{r}\left[\bar{C}_{\mid g}\right], g \in G, t \in \bar{H}\right]$,

(4) $\bar{C}$ is involutive.

Clearly, $(\overline{1}),(\overline{2})$ and $(\overline{4})$ hold. 'To see $(\overline{3})$, take an arbitrary vector $v \in \bar{C}_{1 .}$. we have $v=L_{\mathrm{g}}(w)$ for some $w \in c$. Since $R_{l}(v)=L_{\mathrm{r}}\left(R_{l}(w)\right)$, we need only to observe that $R_{t}(w) \in \bar{C}_{\mid l}$ for $t \in \bar{H}$. Write $t=l i m t_{n,}, t \in H$; then, by the closedness of $\bar{C}$ in $T G$, we obtain that $R_{l}(w)=\lim R_{i_{n}}(w) \in \bar{C}$ because $R_{r, l}[\bar{C}]=\bar{C}$.

Step 2. Let $\bar{C} \subset T G$ be a distribution realizing conditions $(\overline{1}) \div(\overline{4})$ above. Via the epimorphism $\alpha: T G \rightarrow Q$ we define a subbundle $C^{\prime} \subset Q$ by $C_{\mid g}^{\prime}=\alpha_{g}\left[\bar{C}_{\mid g}\right], g \in G$. [The fact that $C^{\prime}$ is a subbundle is obtained from the relation $E \subset \bar{C}$ which holds by $(\overline{2})]$. $C^{\prime}$ fulfils the following conditions:

(1') $Q^{\prime} \oplus C^{\prime}=Q$ where $Q^{\prime}=E_{h} / E \subset Q$

(2') $C^{\prime \prime}$ is $\bar{H}$-right-invariant [i.c. $\left.C_{\mid g t}^{\prime}=\bar{R}_{i}\left[C_{\mid, t}^{\prime}\right], g \in G, t \in \bar{H}\right]$,

(3') $\quad l_{i}(G ; H):=\operatorname{Sec}^{\circ} C^{\prime} \cap /(G ; H)$ is a Lie subalgebra of $/(G ; H)$.

$\left(I^{\prime}\right)$ and $\left(2^{\prime}\right)$ are obvious. To check (3'), take arbitrary $\zeta, \nu \in l_{i}(G ; H)$ and write $\zeta=\bar{X}, \nu=\bar{Y}$ for some vector fields $X, Y \in X(\bar{C})$. According to $(\overline{4})$, $[X, Y] \in X(\bar{C})$, which gives the relation $\overline{[X, Y]} \in \operatorname{Sec} C$. On the other hand (see

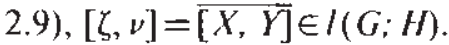

Step 3. Let $C^{\prime} \subset Q$ be any vector subbundle realizing conditions (1') $\div\left(3^{\prime}\right)$ above. Via the linear homomorphism $\beta: Q \rightarrow A(G ; /)$ we define a subbundle $C \subset A(G ; H)$ by $C_{\mid g}=\beta_{g s}\left[C_{\mid s}^{\prime}\right], g \in \pi_{h}{ }^{-1}(\bar{g}), \bar{g} \in G / \bar{H}$. 'l'hanks 10 the equality $\beta, \bar{R}_{1}=\beta, l \in \bar{H}$, the correctness of this delinition is evident. To see that $C$ is a $C^{\infty}$ vector subbundle of $A(G ; H)$, it is sufficient to notice that a local $C^{\infty}$ cross-section of $A(G ; H)$ lying in $C$ and passing through an arbitrarily taken vector from $C$ exists. Let $\bar{v} \in C_{1, g}^{\prime}$ and $\bar{g}=\pi_{h}(g)$. Take a local 
$C^{\infty}$ cross-section $\varphi: U \longrightarrow G$ of the submersion $\pi_{r}: G \rightarrow G / \bar{H}$, such that $\varphi(\bar{g})=g$, and consider the diagram

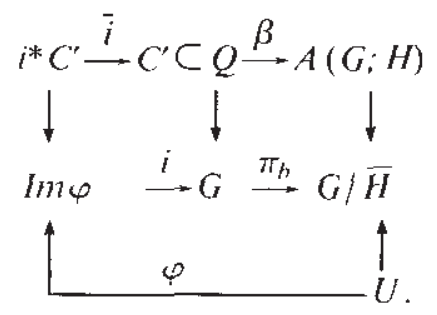

Diminishing $U$ if necessary, we may assume that the vector bundle $i^{*} C^{\prime}-\operatorname{lm} \varphi$ has a global $C^{\infty}$ cross-section $Z$ passing through $\bar{v}$. Put $\xi=$ $\beta " \bar{i} " Z " \varphi: U \longrightarrow A(G ; H) ; \xi$ is, of course, a $C^{\infty}$ cross-section of $A(G ; H)$ over $U$ such that $\xi(\bar{g})=[\bar{v}]$. The vector bundle $C$ fulfils the conditions

(1) $\mathbf{g} \oplus C=A(G ; H)$,

(2) $\operatorname{Sec} C$ is a I.ie subalgebra of $\operatorname{Sec} A(G ; H)$.

(1) is evident by the observation that $\beta_{y}$ maps isomorphically $Q_{1{ }^{\prime \prime}}$ onto $\mathbf{g}_{1 \bar{g}}$. To see (2), take arbitrary $\xi, \eta \in \operatorname{Sec} C$. According to 2.8 , there exist transversal fields $\zeta, v$ such that $c_{\xi}=\xi$ and $c_{p}=\eta$. Of course, $\beta_{5}\left(\zeta_{g}\right)=\xi_{\bar{g}}$ and $\beta_{g}\left(v_{g}\right)=\eta_{g}, g \in \pi_{s}^{-1}(\bar{g})$. From the definition of $C$ we obtain that $\zeta$ and $v$ belong to $l_{i}(G ; H)$. By $(3),\left[\zeta, v^{\prime}\right] \in \operatorname{Sec} C^{\prime \prime} \cap /(G ; /)$, therefore $\|\xi, \eta\|=$ ${ }_{|\zeta . \mathrm{v}|} \in \operatorname{Sec} C$.

Step 4. Let $C \subset A(G ; H)$ be a vector subbundle realizing conditions (1) and (2) above. "Then, of course, a splitting $\lambda$ of the Atiyah sequence of $A(G ; H)$, see the diagram

$$
O \rightarrow \mathbf{g} C A(G: H)=\mathbf{g} \oplus C \frac{\gamma}{\frac{\gamma}{\lambda}} T(G / \bar{H}) \longrightarrow O
$$

such that $I m \lambda=C$, is a flat connection in $A(G ; H)$.

Combining the above theorems we get

3.5. Corollary. The evistence of a Lie subalgebra c C g fulfilling $\mathrm{c}+\overline{\mathrm{h}}=\mathrm{g}$ and $\mathrm{c} \cap \overline{\mathrm{h}}=\mathrm{h}$ implies the trivialit of the Lie algetroid $A(G ; H)$. 


\section{MAIN RESULTS}

Let the symbols $H, \bar{H}, G, \mathrm{~h}, \overline{\mathrm{h}}$. $\mathrm{g}$ have the same meaning as in the previous two sections.

4.1. Theorem. If there is a Lie subalgebra $\subset \subset \mathfrak{g}$ such that (a) $\mathrm{c}+\overrightarrow{\mathrm{h}}=\mathfrak{g}$, (b) $c \cap \overline{\mathrm{h}}=\mathrm{k}$, then the Lie algebra $\mathrm{h}$ is minimally closed.

Proof. Corollary 3.5 states that the Lie algebroid $A(G ; H)$ of the Lie subgroup $H \subset G$ is trivial, i.e. there exists a $L_{\text {Lie }}$ algebroid isomorphism $\Phi: A(G ; H) \rightarrow A_{1}:=T(G / \bar{H}) \times \overline{\mathbf{h}} / \mathrm{h}$. Such a lie algebroid is, of course, integrable: $A_{0}$ is the Lie algebroid of the trivial principal fibre bundle $P=G / \vec{H} \times F$ for an arbitrarily taken Lie group $F$ with the abelian Lie algebra $\bar{h} / \mathrm{h}$, see 1.3 . The following reasoning is due to R. Almeida and $P$. Molino, see the proof of their theorem [13, p. 138]. Consider the Lie algebroid $(7 G \times \overline{\mathrm{h}} / \mathrm{h}$. $\llbracket\left[\cdot, \rrbracket, p r_{1}\right)$ of the trivial principal fibre bundle $G \times F$. The linear homomorphism of vector bundles.

$$
\lambda: T G \rightarrow T G \times \bar{h} / \mathrm{h} . \quad v \rightarrow\left(v, p r_{2} " \Phi([\bar{v}])\right)
$$

is a connection in this Lie algebroid. $\lambda$ is flat. Indeed, it is sufficient to show the equality $\llbracket \lambda X, \lambda Y \rrbracket=\lambda[X, Y]$ only for $X, Y \in X(G)$ such that the corresponding cross-sections $\bar{X}, \bar{Y}$ of $Q$ are transversal fields. However, the equality is then easy to obtain by using the fact that $\Phi$ is a homomorphism of L.ie algebras, namely, writing $\lambda X=\left(X, p r_{2} " \Phi "\left(\bar{X}^{\prime} \pi_{h}\right)\right.$ (and, analogously, for $\lambda \gamma)$, we have

$$
\begin{aligned}
& \llbracket \lambda X, \lambda Y \rrbracket]=\llbracket\left(X, p r_{2} \cdots \Phi \cdots\left(\bar{Y} * \pi_{h}\right),\left(Y, p r_{2} " \Phi \cdots\left(\bar{r} * \pi_{h}\right) \rrbracket\right.\right.
\end{aligned}
$$

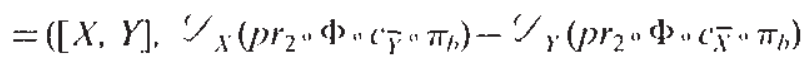

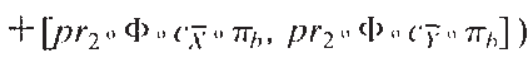

$$
\begin{aligned}
& =\left([X, \eta] \cdot \gamma_{\gamma+\bar{x}}\left(p r_{2} \| \Phi \cdots c_{y}\right) \cdots \pi_{h}-\mathcal{Y}_{\gamma \cdots \bar{y}}\left(p r_{2} \cdots \Phi \cdots(\bar{x}) \cdots \pi_{h}\right.\right. \\
& +\left[p r_{2} \cdots \Phi \cdot c \bar{x}, p r_{2}, \Phi \cdots(\gamma] \cdots \pi_{b}\right)
\end{aligned}
$$

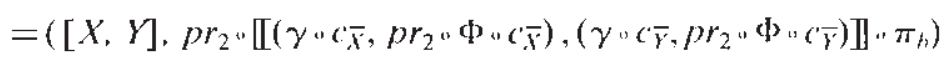

$$
\begin{aligned}
& =\left([X, Y], p r_{2} "[\Phi * c \bar{X}, \Phi \cdot c \cdot \bar{\gamma}] \| \pi_{r}\right) \\
& =\left([X, Y], p r_{2} " \Phi " \llbracket c \bar{x}, c_{Y} \rrbracket " \pi_{h}\right) \\
& =\left([X, Y] \cdot p r_{2} " \Phi "\left(\pi, Y \mid " \pi_{b}\right)\right. \\
& =\lambda[X, Y] \text {. }
\end{aligned}
$$


Let $D$ be the connection in $G \times F$ determined by $\lambda$, i.e. the right-invariant distribution $D \subset T(G \times F)$ for which

$$
D_{\mid(k . \omega)}=\left\{\left(v, p r_{2} " \Phi([\bar{v}]) ; \quad v \in T G\right\}, \quad g \in G,\right.
$$

where $e$ denotes the neutral element of $F$. The flatness of $\lambda$ implies the involutivity of $D$. Consider the diagram

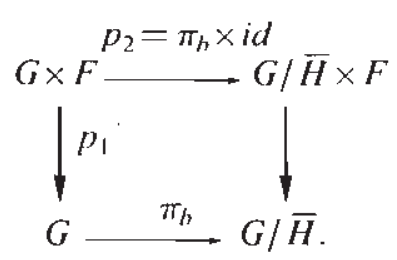

Let $\widetilde{G} \subset G \times F$ be any leaf of the distribution $D$. Of course, $\tilde{p}_{1}=p_{1} \mid \tilde{G}$ : $\tilde{G} \longrightarrow G$ is a covering and, which is easy to obtain, $\tilde{p}_{2}=p_{2} \mid \tilde{G}: \widetilde{G} \rightarrow G / \bar{H} \times F$ is a submersion. Denote by the lifting (by $\tilde{p}_{1}$ ) of the foliation in $\tilde{G}$. l.et $(g, a) \in \bar{G}$. For $v \in T_{g} G$, the following conditions are equivalent:

(1) $v$ is tangent to

(2) $\tilde{p}_{1^{*}(g, a)^{-l}(v) \text { is tangent to }}$

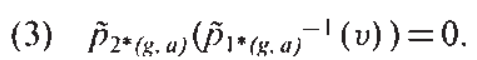

From this we obtain that is defined by the submersion $\tilde{p}_{2}: \widetilde{G} \rightarrow G / \bar{H} \times F$, in particular, the leaves of $\bar{F}$ are closed. Introducing in $\tilde{G}$ a structure of a group in the standard way we obtain: $\tilde{G}$ is a Lie group and $\tilde{D}_{1}$ is a local isomorphism of Lie groups. It is a standard calculation to obtain that $\bar{F}$ is then the foliation of left cosets of $\widetilde{G}$ by $\widetilde{F}$ where $\widetilde{F}$ is a connected Lie subgroup of $\tilde{G}$ with the Lie algebra equalling $\tilde{\mathrm{h}}=\tilde{p}_{1^{*} \tilde{e}^{-1}}[\mathrm{~h}]$ ( $\tilde{e}$ being the neutral element of $\tilde{G}$ ). Therefore $\tilde{F}$ is a closed Lie subgroup. Of course, $F$, being closed alter the lifting to some covering, is also closed after lifting it to the universal one, which means that $\mathrm{h}$ is minimally closed.

4.2. Theorem. If $\pi_{1}(G)$ is finite and $H \neq \bar{H}$, then there exists no lie subalgebra $c \subset$ fulfilling the conditions $\mathrm{c}+\overline{\mathrm{h}}=\mathrm{g}$ and $\mathrm{c} \cap \overline{\mathrm{h}}=\mathrm{h}$.

Proof. Let $\pi_{1}(G)$ be finite. Then, the universal covering is finite, which implies the nonclosedness of the lifting $\tilde{H}$ of $H$. Our assertion follows now trivially from the previous theorem.

To finish with, we can ask 


\section{Can Theorem 4,1 be inverted?}

It turns out that the answer is no.

4.3. Example. Let $G=U(2)$. Suppose that $\bar{H}:=T$ is a maximal torus in $G$. It is well known that $\operatorname{dim} T=2$ and the lifting of $T$ to the universal covering $\mathbf{R} \times S U(2) \longrightarrow U(2)$ is isomorphic to the cylinder $\mathbf{R} \times S^{\prime}$. Therefore, any Lie subalgebra $h$ of $\bar{h}(\bar{h}-$ the Lie algebra of $\vec{H})$ is minimally closed. We prove, using theorem 0.2 , that there exists some 1 -dimensional Lie subalgebra $\mathbf{k}$ of $\overline{\mathbf{h}}$ for which.

(i) no Lie subalgebrat $c \subset g$ fulfilling (a) and (b) from 0.1 exists.

(ii) the corresponding connected Lie subgroup of 7 is dense in $T$.

Let $h_{p}: V\left(\overline{\mathrm{k}}^{*}\right) \longrightarrow H_{\mathrm{d} \mathrm{R}}(G / T)$ be the Chern-Weil homomorphism of the $T$-principal fibre bundle $P=(G \rightarrow G / T) . G$ and $T$ have the same rank, therefore, according to $[3 ;$ Th.VII, p. 467], we have that

$$
h_{p}^{(2)}: \overline{\mathbf{h}}^{*} \longrightarrow H_{\mathrm{d} \mathbf{R}}^{2}(G / T)
$$

is surjective. Moreover, $\operatorname{dim} \overline{\mathrm{h}}^{*}=2$ and $\operatorname{dim} H_{\mathrm{d} \mathbf{R}}^{2}(G / T)=1$, thus $\operatorname{dim} \operatorname{Kerh}_{\mathrm{P}}^{(2)}=1$. Then it is obvious that there exists a covector $O \neq \beta \in \bar{h}^{*}$ such that (1) $h_{p}^{(2)}(\beta) \neq 0,(2) \mathbf{h}:=\operatorname{Ker} \beta \subset \overline{\mathbf{h}}$ is a subspace such that the corresponding Lie subgroup $H \subset T$ is dense in $T$. Of course, the superposition

$$
(\overline{\mathrm{h}} / \mathrm{h})^{*} \stackrel{j}{\rightarrow} \overline{\mathrm{h}}^{*} \stackrel{h_{P}^{(2)}}{\rightarrow} H_{\mathrm{dR}}^{2}(G / T)
$$

is nontrivial: $h_{P}^{(2)}, j(\bar{\beta}) \neq 0$ where $\bar{\beta} \in(\overline{\mathbf{h}} / \mathbf{h})^{*}$ is a linear homomorphism determined by $\beta$. Theorem 0.2 implies the nonexistence of a Lie subalgebra $c \subset \mathfrak{g}$ fulfilling (a) and (b) above.

\section{References}

[1] R. Almingn, P. Molino: Suites d'Atiyah et feuilletages transversallement complets, C. R. Acad. Sci. Paris Ser. I Math., 300 (1985), 13-15.

[2] - Suites d'Atiyah, feuilletages et quantification geometrique, Lstrait du Seminaire du Geometrie differentielle, Montpellier, 1984-85.

[3] W. GREUB, S. HALPERIN, R, VANSTONE: Connections, Curvature, and Cohomology, Vol. III. Academic Press, New York and London, 1976.

[4] J. KUBARSKI: Exponential mapping for Lie groupoids, Colloq. Marh. XI.VIl (1982), 267-282. 
[5] - Lic algebroid of a principal libre bundle, Publications da Deparment de Mathematiques de L'Universite de LYON I. in printing.

[6] - Lie algebroid of a principal fibre bundle - three equivalent definitions, Prace Nalkowe Politechniki Szczecinskiej, 11 (1988), 123-145.

[7] - The Chern-Weil homomorphism of regular Lie algebroids and TCfoliations. Preprim No 9. Institute of Mathematics, Technical University of l_ódí, November 1988.

[8] - Pontryagin algebra of a transitive Iie algebroid, Supplemento ai Rendiconti del Circolo Matematico di Palermo, Proceedings of the Winter School on Geometry and Physics. SRNI. 9-16 January, 1988 (in printing).

[9] K. MnCKEN7.II: Lie groupoids and Lie algebroids in differemial Geomem. Cambridge, 1987.

[10] - A note on L.ic algebroids which arisc from groupoid actions, Cahiers de Topologic et Geometrie Differentielle Categoriques, XXVIII-4 (1987), 283-302.

[11] A. MAICEV: Subgroups of Lic groups in the large, $C$. R. (Doklady) Acad. Sit. URSS (N. S.) 36 (1942), 5-7 (Kraus Reprint LTD. VADUZ 1963).

[12] P. Morino: Etude des feuilletages transversalement complets et applications, Atrh. Sci. Erole Norm. Sup., 10(3) (1977), 289-307.

[13] - Ricmannian Foliations, Progress in Mathemarics Vol. 73, Birkhäuser Boston Basel. 1988.

[14] NGO-VAN-QuE: Sur l'espace de prolongement differentiable, J, Differential Geom. 2.1. (1968), 33-40.

[15] J. PRADINES: Theoric de Lie pour les groupoides differentiables dans la categorie des groupoides. Calcul differential dans la categorie des groupoides infinitesimaux. C, R. Acad. Sci. Ser. A-B, Paris. 264 (1967), 265-248.

[16] - : Theorie de L.ie pour les groupoides differentiables. Ati Conv Intern Geom 7 Difr. Bologna, 1967, Bologna-Amsterdam.

[17] E. WINBlERG, A. ONISHCHIK: A seminar on Lie grotes and algebraic groups (in Russian), Moscov. 1988. 